\title{
A prediction model using machine-learning algorithm for assessing intrathecal hyperbaric bupivacaine dose during cesarean section
}

\author{
Chang-na Wei ${ }^{1 \dagger}$, Li-ying Wang ${ }^{1 \dagger}$, Xiang-yang Chang ${ }^{1}$ and Qing-he Zhou ${ }^{2^{*}}$ (D)
}

\begin{abstract}
Background: The intrathecal hyperbaric bupivacaine dosage for cesarean section is difficult to predetermine. This study aimed to develop a decision-support model using a machine-learning algorithm for assessing intrathecal hyperbaric bupivacaine dose based on physical variables during cesarean section.

Methods: Term parturients presenting for elective cesarean section under spinal anaesthesia were enrolled. Spinal anesthesia was performed at the L3/4 interspace with $0.5 \%$ hyperbaric bupivacaine at dosages determined by the anesthesiologist. A spinal spread level between T4-T6 was considered the appropriate block level. We used a machine-learning algorithm to identify relevant parameters. The dataset was split into derivation (80\%) and validation (20\%) cohorts. A decision-support model was developed for obtaining the regression equation between optimized intrathecal $0.5 \%$ hyperbaric bupivacaine volume and physical variables.

Results: A total of 684 parturients were included, of whom 516 (75.44\%) and 168 (24.56\%) had block levels between T4 and T6, and less than T6 or higher than T4, respectively. The appropriate block level rate was 75.44\%, with the mean bupivacaine volume $[1.965,95 \% \mathrm{Cl}(1.945,1.984)] \mathrm{ml}$. In lasso regression, based on the principle of predicting a reasonable dose of intrathecal bupivacaine with fewer physical variables, the model is " $Y=0.5922+$ $0.055117^{*} X_{1}-0.017599^{*} X_{2}{ }^{\prime \prime}\left(Y\right.$ : bupivacaine volume; $X_{1}$ : vertebral column length; $X_{2}$ : abdominal girth), with $\lambda$ 0.055, MSE 0.0087, and $R^{2} 0.807$.

Conclusions: After applying a machine-learning algorithm, we developed a decision model with $R^{2} 0.8070$ and MSE due to error 0.0087 using abdominal girth and vertebral column length for predicting the optimized intrathecal $0.5 \%$ hyperbaric bupivacaine dosage during term cesarean sections.
\end{abstract}

Keywords: Machine learning algorithm, Bupivacaine dosage, Cesarean section, Spinal anesthesia, Physical variables

\footnotetext{
* Correspondence: jxxmxy@163.com

${ }^{+}$Chang-na Wei and Li-ying Wang contributed equally to this work.

${ }^{2}$ Department of Anesthesia, Affiliated Hospital of Jiaxing University, Jiaxing, Zhejiang Province, China

Full list of author information is available at the end of the article
}

(C) The Author(s). 2021 Open Access This article is licensed under a Creative Commons Attribution 4.0 International License, which permits use, sharing, adaptation, distribution and reproduction in any medium or format, as long as you give appropriate credit to the original author(s) and the source, provide a link to the Creative Commons licence, and indicate if changes were made. The images or other third party material in this article are included in the article's Creative Commons licence, unless indicated otherwise in a credit line to the material. If material is not included in the article's Creative Commons licence and your intended use is not permitted by statutory regulation or exceeds the permitted use, you will need to obtain permission directly from the copyright holder. To view a copy of this licence, visit http://creativecommons.org/licenses/by/4.0/ The Creative Commons Public Domain Dedication waiver (http://creativecommons.org/publicdomain/zero/1.0/) applies to the data made available in this article, unless otherwise stated in a credit line to the data. 


\section{Background}

Cesarean delivery is routinely performed under spinal anesthesia with hyperbaric bupivacaine [1-3]. Bupivacaine provides an appropriate duration of anesthesia to perform cesarean delivery, and hyperbaric bupivacaine may ensure a more predictable block [4]. However, it is still a challenge for the anesthetist to achieve the optimal spinal spread for cesarean delivery [5]. Parturient variables, including age, height, weight, and body mass index (BMI) have been used to predict spinal spread, but results have been inconsistent $[6,7]$.

Previous studies demonstrated that abdominal girth and vertebral column length correlated favorably with spread of intrathecal bupivacaine in term parturients $[8,9]$. Furthermore, an elemental regression equation was established between parturient vertebral column length, abdominal girth, and $0.5 \%$ hyperbaric intrathecal bupivacaine volume for T5 block level [10]. However, the sample size in these studies was relatively small and the accuracy of the regression equation needed further verification $[8,10]$.

In recent years, there has been an advance in machine-learning algorithms in several fields including anesthesiology, which allowed large amounts of data for the development of robust predictive analytics [11-14]. These were was used to predict, interalia, postinduction hypotension [14], intraoperative hypotension [12], and length of hospital stay [15]. In our hospital, more than 1000 parturient women undergo cesarean section under spinal anesthesia annually. The purpose of this prospective study was to develop a more precise decisionsupport model based on more sample size using a machine-learning algorithm for assessing intrathecal hyperbaric bupivacaine dosage based on their physical variables at cesarean section.

\section{Methods}

\section{Patients}

The study protocol was approved by the Ethical Committee of Jiaxing Maternity and Child Health Care Hospital in May 2015 (2015-5) and pre-registered at http://www.chictr.org.cn/ index.aspx (ChiCTR-OOC16009149) on September 3, 2016. The signed informed consent was obtained from all participants. In this prospective observational study, 684 term parturient women who presented for elective cesarean section under spinal anesthesia were enrolled from October 2016 to November 2019. Inclusion criteria were American Society of Anesthesiologists (ASA) physical status II or III, age between 21 and 40 years, and gestational age over 37 completed weeks. Exclusion criteria were patient refusal, significant medical or obstetric comorbidities, multiple pregnancies, failed spinal anesthesia, contraindication to spinal anesthesia, discrepancy in spread between both midclavicular lines, or history of allergy to bupivacaine.

\section{Spinal anaesthesia}

The parturient fasted for $8 \mathrm{~h}$ and received $8 \mathrm{~mL} / \mathrm{kg}$ Ringer's lactate solution via peripheral intravenous access before spinal anesthesia. After entry to the operation room, the standard ASA monitoring was performed. Before spinal puncture, the L3/4 interspace was confirmed by ultrasonic imaging. Spinal anesthesia was performed at the L3/4 interspace in the left lateral decubitus position using a $25 \mathrm{G}$ pencil-point spinal needle (Zhejiang Sujia Medical Equipment Co., Ltd) with midline approach; $0.5 \%$ hyperbaric bupivacaine (Shanghai Hefeng Pharmaceutical Co., Ltd) was injected into the subarachnoid space over $10 \mathrm{~s}$ after a free flow of cerebrospinal fluid had been obtained. The volume of $0.5 \%$ hyperbaric bupivacaine administered was based on the experience of the anesthesiologist. After these procedures, the woman was then rapidly placed in a supine position, with a right pelvic wedge placed to facilitate left uterine displacement. All anesthesia procedures were performed by the same attending physician, and the assessment of cephalad spread of spinal anesthesia was completed by another anesthetist who was blind to the measurement information about the woman. The spinal spread was assessed in both midclavicular lines by an 18-gauge needle for loss of pinprick discrimination 15 min after intrathecal injection and then surgery commenced. If the parturient complained of severe pain (general Visual Analogue Scale $[\mathrm{VAS}] \geq 4$ ) during surgery, remifentanil was administered with micropump injection for rescue analgesia. If, based on the assessment of the anesthetist, the woman could not endure the surgery under spinal anesthesia, general anesthesia was performed. Hypotension was defined as systolic pressure values $<90 \mathrm{mmHg}$ or systolic pressure decreases $>30 \%$ and was treated with $6 \mathrm{mg}$ of ephedrine or $100 \mu \mathrm{g}$ of phenylephrine intravenously. Bradycardia was defined as heart rate values $<55$ beats/min and was treated with atropine $0.5 \mathrm{mg}$ intravenously.

\section{Outcome measures}

Abdominal girth was measured at the level of the umbilicus at the end of expiration when the parturient was placed supinely on the horizontal operating table. Vertebral column length was measured from the C7 vertebra to the sacral hiatus $(\mathrm{C} 7-\mathrm{SH})$. Cephalad spread of spinal anesthesia was assessed by testing for loss of pinprick discrimination in both midclavicular lines at 3 min intervals after intrathecal injection. The spread level at 15 min after intrathecal bupivacaine injection was used for the analysis. The spinal spread level between T4-T6 was considered the appropriate block level. The volume of intrathecal bupivacaine was recorded. Parturient demographic variables, including age, height, fundal height, 
and weight were recorded. Demographic variables, such as fetal biparietal diameter, were also recorded.

As predictors for the machine-learning models, we included parturient age, height, fundal height, weight, abdominal girth, vertebral column length, and fetal biparietal diameter. The primary outcome was to obtain the regression equation between optimized intrathecal $0.5 \%$ hyperbaric bupivacaine dosage and physical variables.

\section{Decision-support model analysis}

Machine-learning algorithm was performed by Python 3.7.1. The objective function of lasso regression is:

$$
\min _{\omega}\|y-X \omega\|_{2}^{2}+\lambda\|\omega\|_{1}
$$

In the function, $\lambda$ is a parameter that controls the complexity of the model. With lasso regression, controlling the parameter of $\lambda$ can result in a sparse solution: the coefficient of unimportant features will be assigned to 0 , and the important features will be highlighted in order according to the weight value so as to achieve the importance ranking of features, and further control the complexity of the algorithm according to the selected input features.

Therefore, based on the lasso regression algorithm, our study explored the relationship between the seven individual physical variables listed above and bupivacaine dosage, then obtained the regression equation between optimized intrathecal $0.5 \%$ hyperbaric bupivacaine dosage and the physical variables.

The statistical model strives to predict an intrathecal bupivacaine dosage with fewer physical variables. $R^{2}$ is the determination coefficient, which is the degree of fitting for the obtained regression equation. Meansquare error (MSE) is a single value that provides information about the goodness fit of the regression line. The smaller the MSE value, the better the fit, as smaller values imply smaller magnitudes of error. The data set (term parturient with appropriate block level) was split into derivation $(80 \%)$ and validation $(20 \%)$ cohorts. The derivation cohort parturients were used to derive for the prediction model, and the validation cohorts were used to validate the model. We gradually increased the number of independent variables from 2 to 5 (physical variables), optimized the $\lambda$ value, and balanced the value of $\mathrm{R}^{2}$, MES, and the number of independent variables.

All statistical analyses were performed with SPSS version 19 (IBM Corp). We presented the data as means \pm standard deviations (SDs) or numbers (\%) as appropriate. We tested quantitative data by using the independent $t$-test. A two-tailed $p$ value $<0.05$ denoted statistical significance.

\section{Results}

A total of 684 parturients were included in this study, of whom 516 had a block level between T4 and T6 (appropriate block level) and 168 had a block level lower than T6 or higher than T4 (inappropriate block level). The appropriate block level rate was $75.44 \%$, with the mean bupivacaine volume $[1.965,95 \% \mathrm{CI}(1.945,1.984)] \mathrm{ml}$. The spread level of 69 parturients was less than T6 and 99 parturients higher than T4. Compared with those with appropriate block levels, weight and abdominal girth were greater in the group with inappropriate block levels $(p<0.05$ and $p<0.01$, respectively); there was no obvious difference in bupivacaine dosage and other physical variables in those women with appropriate and inappropriate block levels (Table 1).

A total of 412 term parturients were used to derive for the prediction model. In lasso regression, when the physical variables included in the equation increased from 2 to $5, \mathrm{R}^{2}$ increased from 0.8070 to 0.81325 , and MSE decreased from 0.0087 to 0.00844 , both with little changed.

On decision tree analysis, vertebral column length and abdominal girth were the top two physical performance variables with respect to the dosage of intrathecal bupivacaine; the model is " $\mathrm{Y}=0.5922+0.055117 " \mathrm{X}_{1}$ $0.017599 * \mathrm{X}_{2}$ " $\left(\mathrm{Y}: 0.5 \%\right.$ bupivacaine volume; $\mathrm{X}_{1}$ : vertebral column length; $\mathrm{X}_{2}$ :abdominal girth), with the $\lambda 0.055$, MSE 0.0087 and $R^{2} 0.807$ (Table 2). The remaining 104 participants were used to validate the model. The actual bupivacaine volume was $1.94 \pm 0.21 \mathrm{~mL}$, with a predicted bupivacaine volume of $1.95 \pm 0.19 \mathrm{~mL}(p>0.05) ; \mathrm{R}^{2}$ of all women during the validation was above 0.8 , indicating that the model was reliable (Figs. 1 and 2).

In the current study, 282 parturients $(41.23 \%)$ were found to develop hypotension during cesarean section and were treated with ephedrine and/or norepinephrine.

\section{Discussion}

In this prospective, observational study, after applying a machine-learning algorithm, we developed the decision model: $\mathrm{Y}=0.5922+0.055117^{*} \mathrm{X}_{1}-0.017599^{*} \mathrm{X}_{2}$ (Y: $0.5 \%$ hyperbaric bupivacaine volume; $\mathrm{X}_{1}$ : vertebral column length; $X_{2}$ : abdominal girth), with the $\lambda 0.055$, MSE 0.0087 , and $R^{2} 0.807$. On decision-tree analysis, vertebral column length and abdominal girth were the top two performance physical variables with respect to intrathecal bupivacaine dosage.

According to the data type of this study, multiple linear regression, ridge regression, and lasso regression are usually used for data analysis. Multiple linear regression has the characteristics of simple construction, easy implementation, and low operation complexity, but the model is prone to overfitting. To control the complexity of the model, penalties or other constraints are often 
Table 1 Clinical characteristics of term parturients

\begin{tabular}{lllll}
\hline & Overall & Appropriate block & Inappropriate block & $P$ value \\
\hline No. pts. (\%) & 684 & $516(75.44 \%)$ & $168(24.56 \%)$ & $30.8 \pm 4.6$ \\
Age, yrs & $30.7 \pm 4.5$ & $30.7 \pm 4.5$ & $71.0 \pm 10.1$ & 0.717 \\
Weight, kg & $69.5 \pm 9.6$ & $69.1 \pm 9.4$ & $159.1 \pm 4.8$ & 0.022 \\
Height, cm & $159.3 \pm 4.7$ & $159.4 \pm 4.6$ & $56.4 \pm 3.8$ & 0.405 \\
Vertebral column length, cm & $56.6 \pm 3.2$ & $56.7 \pm 3.0$ & $101.2 \pm 7.7$ & 0.323 \\
Abdominal girth, cm & $99.9 \pm 7.1$ & $99.5 \pm 6.9$ & $35.2 \pm 2.6$ & 0.007 \\
Fundal height, cm & $35.2 \pm 2.8$ & $35.2 \pm 2.9$ & $9.2 \pm 0.4$ & 0.789 \\
Fetal biparietal diameter, cm & $9.1 \pm 0.5$ & $9.1 \pm 0.5$ & $3.4 \pm 0.6$ & 0.076 \\
Fetal weight, kg & $3.3 \pm 0.7$ & $3.4 \pm 0.7$ & $2.0 \pm 0.1$ & 0.445 \\
Bupivacaine dosage, $\mathrm{mL}$ & $2.0 \pm 0.2$ & $2.0 \pm 0.2$ & 0.057 \\
\hline
\end{tabular}

added to the model; that is, regularization techniques. Ridge regression and lasso regression are currently popular regularization regression techniques. The goal of the two is the same; that is, to minimize the sum of squared residuals, but the constraints on the regression coefficients are different. Ridge regression can effectively solve the problem of overfitting but, as the value changes, its feature coefficients become uniformly small, making it impossible to discern the importance of each feature. Lasso regression, however, effectively solves the overfitting problem; it can obtain a sparse solution by controlling the parameters: the coefficients of unimportant features will be assigned to 0 , and the important features will be highlighted in order according to the weight value, so as to achieve the importance ranking of features, and further control the complexity of the algorithm according to the selected input features.
In the present study, according to the results of parameter selection of lasso regression and model evaluation for different parameters, when the physical variables included in the equation increased from 2 to 5 , MSE and $R^{2}$ were not obviously increased. On decisiontree analysis, vertebral column length and abdominal girth were the top two performance physical variables with respect to intrathecal bupivacaine dosage. Over one hundred term parturients were used to validate the model, and $\mathrm{R}^{2}$ of all patients during the validation was above 0.8 , indicating that the model was reliable.

Previous studies reported that the median satisfactory block height for the loss of pinprick discrimination during spinal anesthesia for cesarean section was $\mathrm{T} 5$, and the interquartile range (IQR) was from T4-T6 [16]. In our previous study, we set $\mathrm{T} 5$ as the appropriate spinal spread level and found the vertebral column length and abdominal girth to be the top two performance physical

Table 2 Parameter selection of lasso regression and model evaluation for different parameters

\begin{tabular}{|c|c|c|c|c|c|}
\hline$\lambda$ Value & Selected physical variable & Weight for variable & Equation Intercept & MSE & $R^{2}$ \\
\hline \multirow[t]{2}{*}{0.055} & Abdominal girth & -0.017599 & 0.5922 & 0.0087 & 0.8070 \\
\hline & Vertebral column length & 0.055117 & & & \\
\hline \multirow[t]{3}{*}{0.038} & Height & 0.000141 & 0.5071 & 0.0086 & 0.8108 \\
\hline & Abdominal girth & -0.018174 & & & \\
\hline & Vertebral column length & 0.057225 & & & \\
\hline \multirow[t]{4}{*}{0.031} & Height & 0.000286 & 0.4516 & 0.0085 & 0.81199 \\
\hline & Weight & -0.000171 & & & \\
\hline & Abdominal girth & -0.018232 & & & \\
\hline & Vertebral column length & 0.058107 & & & \\
\hline \multirow[t]{5}{*}{0.020} & Height & 0.000618 & 0.3687 & 0.00844 & 0.81325 \\
\hline & Weight & -0.000600 & & & \\
\hline & Fundal height & -0.001253 & & & \\
\hline & Abdominal girth & -0.017910 & & & \\
\hline & Vertebral column length & 0.059371 & & & \\
\hline
\end{tabular}




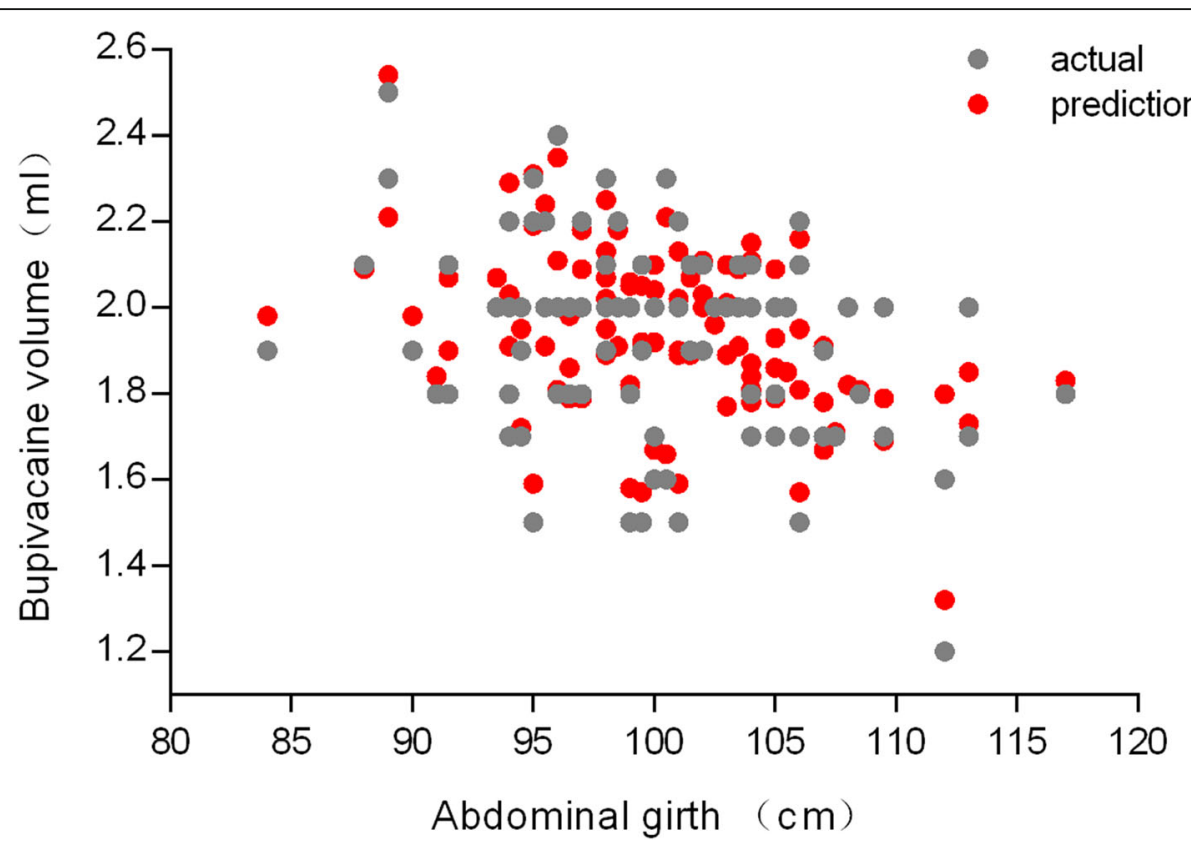

Fig. 1 Actual and predicted bupivacaine volume for validation cohorts with 104 term parturients for validation; the ordinate is bupivacaine volume and abscissa is abdominal girth

variables [10]. In the current study, we set appropriate spinal spread levels for the loss of pinprick discrimination as T6, T5, and T4 at 15 min after intrathecal injection, and also found vertebral column length and abdominal girth to be the top two performance physical variables. We need to note that the regression model obtained in this study is more clinically valuable.

In current study, the appropriate block level rate was $75.44 \%$, with the mean bupivacaine volume $1.965 \mathrm{ml}$ $(9.825 \mathrm{mg})$. The mean bupivacaine dose was slightly

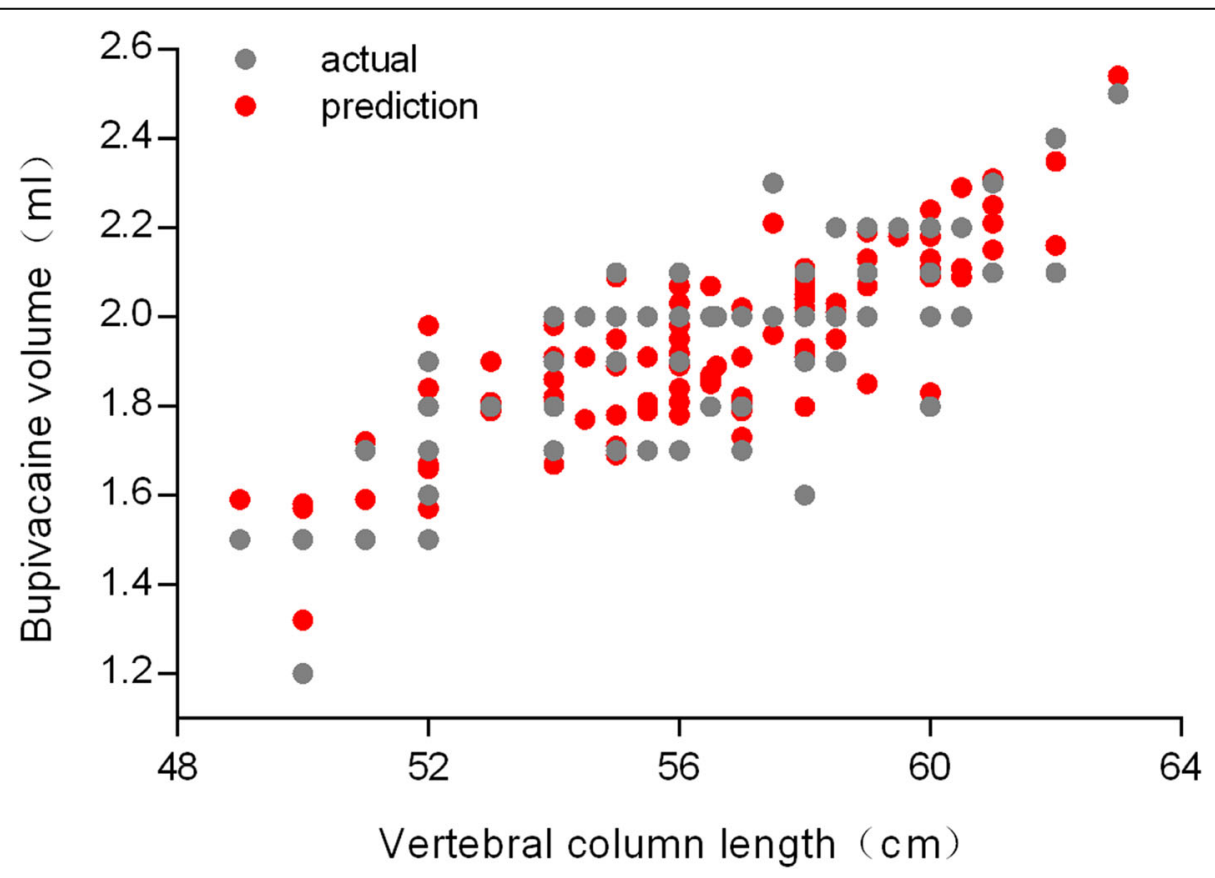

Fig. 2 Actual and predicted bupivacaine volume for validation cohorts with 104 term parturients for validation; the ordinate is bupivacaine volume and abscissa is vertebral column length 
lower than previous study reported that bupivacaine provides anesthesia in almost all patients (ED95) at doses that range between 11 and $13 \mathrm{mg}$ [17]. It may be that the study subjects only included Asians.

Studies have proven that, in pregnant women, soft tissues may migrate inward into the vertebral canal [18], and engorgement of the extradural venous plexus occurs when pregnant women are in the supine position because of obstruction of the inferior vena cava by the enlarged uterus $[19,20]$. Thus, an increased abdominal girth in the parturient causes a decrease in lumbosacral cerebrospinal fluid volume. Carpenter et al. [21]. reported that lumbosacral cerebrospinal fluid volume is the main determinant of the spread of spinal anesthesia. Our recent study showed that abdominal girth and dorsosacral distance were correlated with lumbosacral cerebrospinal fluid volume [22]. Therefore, the maternal abdominal girth and vertebral length may have a predictive effect on the spinal spread due to their effect on the volume of the lumbosacral cerebrospinal fluid.

Previous studies reported incidences of hypotension varying from 1.9 to $71 \%[23,24]$. In the present study, hypotension during cesarean section occurred in $41.23 \%$ of participants and was treated with ephedrine and/or norepinephrine. Most of these patients were term parturients with appropriate spinal spread levels. Thus, when performing a cesarean section under spinal anesthesia, we must strictly monitor the patient's hemodynamic status, irrespective of whether or not the anesthesia block level is in the appropriate range.

This study has several limitations. First, only the spread level at $15 \mathrm{~min}$ after intrathecal bupivacaine injection was used for the analysis. We know that the spinal spread changes over time. Second, intrathecal hyperbaric bupivacaine with opioid was not studied in current study and it is worthy of further study. Third, the model does not take into account the multitude of other factors, such as drug factors, position factors, surgical factors and so on. Forth, this model is based on Asians, and whether it is accurate in other races requires further research. Despite these limitations, the current machine-learning algorithm provides new insights into the potential impact of controversial parameters.

\section{Conclusion}

In conclusion, in the current study, after applying a machine-learning algorithm, we developed a decision model with the coefficient of determination 0.807 and the mean of squares due to error 0.0087, using two physical variables for predicting the intrathecal $0.5 \%$ hyperbaric bupivacaine dosage during cesarean section in term parturients. Among the parturient physical variables, abdominal girth and vertebral column length were the two most significant factors, which can be used for predicting the intrathecal bupivacaine dosage during cesarean section.

\section{Abbreviations}

MSE: Mean-square Error; $R^{2}$ : Coefficient of determination; BMl: body mass index; ASA: American Society of Anesthesiologists; VAS: Visual Analogue Scale; SPSS: Statistic Package for Social Science; IQR: Inter quartile range

\section{Supplementary Information}

The online version contains supplementary material available at https://doi. org/10.1186/s12871-021-01331-8.

Additional file 1: Figure S1. Visual analysis diagram of correlation between physical variables and intrathecal bupivacaine dose for the parturients with block levels between T4 and T6. Figure S2. Correlation analysis diagram between physical variables and intrathecal bupivacaine dose for the parturients with block levels between T4 and T6.

\section{Acknowledgments}

We would like to thank Ying-chun Ren for the analysis of data and Wei Deng for discussion of the planning in current study. The study protocol was preregistered at http://www.chictr. org.cn/index.aspx (ChiCTR-OOC-16009149) on September 3, 2016

\section{Authors' contributions}

WCN. Role: This author helped to conduct the study and write the manuscript. WLY. Role: This author helped to conduct the study. CXY. Role: This author helped to conduct the study. ZQH. Role: This author helped to design the study, analyze the data and write the manuscript. All authors have read and approved the manuscript.

\section{Funding}

The study was funded by the Technology Bureau of Jiaxing, Zhejiang Province (2015C23023) and the Key Discipline established with Zhejiang Province and Jiaxing City jointly (2019-ss-ttyx) in the data collection and analysis.

\section{Availability of data and materials}

The datasets used and/or analyzed during the current study are available from the corresponding author on reasonable request (Email: jxxmxy@163. com).

\section{Declarations}

\section{Ethics approval and consent to participate}

The study protocol was approved by the Ethical Committee of Jiaxing Maternity and Child Health Care Hospital in May 2015 (2015-5). The signed informed consent was obtained from all participants.

Consent for publication

Not applicable.

\section{Competing interests}

The authors declare that they have no competing interests.

\section{Author details}

${ }^{1}$ Department of Anesthesia, Jiaxing University Affiliated Women and Children Hospital, Jiaxing, Zhejiang Province, China. ${ }^{2}$ Department of Anesthesia,

Affiliated Hospital of Jiaxing University, Jiaxing, Zhejiang Province, China.

Received: 19 September 2020 Accepted: 31 March 2021

Published online: 14 April 2021

\section{References}

1. Norris MC. Patient variables and the subarachnoid spread of hyperbaric bupivacaine in the term parturient. Anesthesiology. 1990;72(3):478-82. https://doi.org/10.1097/00000542-199003000-00015.

2. Norris MC. Height, weight, and the spread of subarachnoid hyperbaric bupivacaine in the term parturient. Anesth Analg. 1988;67(6):555-8.

3. Russell IF, Holmqvist EL. Subarachnoid analgesia for caesarean section. A double-blind comparison of plain and hyperbaric $0.5 \%$ bupivacaine. $\mathrm{Br}$ J Anaesth. 1987;59(3):347-53. https://doi.org/10.1093/bja/59.3.347. 
4. Vercauteren MP, Coppejans HC, Hoffmann VL, Saldien V, Adriaensen HA. Small-dose hyperbaric versus plain bupivacaine during spinal anesthesia for cesarean section. Anesth Analg. 1998;86(5):989-93. https://doi.org/10.1213/ 00000539-199805000-00014.

5. Hocking G, Wildsmith JA. Intrathecal drug spread. Br J Anaesth. 2004;93(4): 568-78. https://doi.org/10.1093/bja/aeh204.

6. Ekeløf NP, Jensen E, Poulsen J, et al. Weight gain during pregnancy does not influence the spread of spinal analgesia in the term parturient. Acta Anaesthesiol Scand. 1997;41(7):884-7. https://doi.org/10.1111/j.1399-6576.1 997.tb04804.x

7. Harten JM, Boyne I, Hannah P, Reinstrup P. Effects of a height and weight adjusted dose of local anaesthetic for spinal anaesthesia for elective caesarean section. Anaesthesia. 2005;60(4):348-53. https://doi.org/10.1111/ j.1365-2044.2005.04113.x.

8. Wei CN, Zhang YF, Xia F, Wang LZ, Zhou QH. Abdominal girth, vertebral column length and spread of intrathecal hyperbaric bupivacaine in the term parturient. Int J Obstet Anesth. 2017;31:63-7. https://doi.org/10.1016/j. ijoa.2017.02.002.

9. Siddiqui KM, Ali MA, Ullah H. Comparison of spinal anesthesia dosage based on height and weight versus height alone in patients undergoing elective cesarean section. Korean J Anesthesiol. 2016;69(2):143-8. https://doi.org/10.4 097/kjae.2016.69.2.143

10. Wei $C N$, Zhou $\mathrm{QH}$, Wang $\mathrm{LZ}$. Abdominal girth and vertebral column length aid in predicting intrathecal hyperbaric bupivacaine dose for elective cesarean section. Medicine (Baltimore). 2017;96:e7905.

11. Gambus $\mathrm{PL}$, Jaramillo S. Machine learning in anaesthesia: reactive, proactive ... predictive! Br J Anaesth. 2019;123(4):401-3. https://doi.org/10.1016/j.bja.2 019.07.009.

12. Wijnberge $M$, Geerts BF, Hol L, Lemmers N, Mulder MP, Berge P, et al. Effect of a machine learning-derived early warning system for intraoperative hypotension vs standard care on depth and duration of intraoperative hypotension during elective noncardiac surgery: the HYPE randomized clinical trial. JAMA. 2020;323(11):1052-60. https://doi.org/10.1001/jama.2020. 0592.

13. Kendale S, Kulkarni P, Rosenberg AD, Wang J. Supervised machine-learning predictive analytics for prediction of Postinduction hypotension. Anesthesiology. 2018;129(4):675-88. https://doi.org/10.1097/ALN. 0000000000002374

14. Hashimoto DA, Witkowski E, Gao L, Meireles O, Rosman G. Artificial intelligence in anesthesiology: current techniques, clinical applications, and limitations. Anesthesiology. 2020;132(2):379-94. https://doi.org/10.1097/ALN. 0000000000002960.

15. Gabriel RA, Sharma BS, Doan CN, Jiang X, Schmidt UH, Vaida F. A predictive model for determining patients not requiring prolonged hospital length of stay after elective primary Total hip Arthroplasty. AnesthAnalg. 2019;129(1): 43-50.

16. Ousley R, Egan C, Dowling K, Cyna AM. Assessment of block height for satisfactory spinal anaesthesia for caesarean section. Anaesthesia. 2012; 67(12):1356-63. https://doi.org/10.1111/anae.12034.

17. Benhamou D, Wong C. Neuraxial anesthesia for cesarean delivery: what criteria define the "optimal" technique? AnesthAnalg. 2009:109(5):1370-3.

18. Hirabayashi $Y$, Shimizu R, Fukuda H, Saitoh K, Igarashi T. Soft tissue anatomy within the vertebral canal in pregnant women. Br J Anaesth. 1996;77(2):1536. https://doi.org/10.1093/bja/77.2.153.

19. Igarashi T, Hirabayashi Y, Shimizu R, Saitoh K, Fukuda H, Suzuki H. Thefiberscopic findings of the epidural space in pregnant women. Anesthesiology. 2000;92(6):1631-6. https://doi.org/10.1097/00000542-2 00006000-00021.

20. Takiguchi T, Yamaguchi S, Tezuka M, Furukawa N, Kitajima T. Compression of the subarachnoid space by the engorged epidural venous plexus in pregnant women. Anesthesiology. 2006;105(4):848-51. https://doi.org/10.1 097/00000542-200610000-00033.

21. Carpenter RL, Hogan QH, Liu SS, Crane B, Moore J. Lumbosacral cerebrospinal fluid volume is the primary determinant of sensory block extent and duration during spinal anesthesia. Anesthesiology. 1998;89(1):249. https://doi.org/10.1097/00000542-199807000-00007.

22. Zhou QH, Shen C, Chen G. Abdominal girth and dorso-sacral distance can be used to estimate lumbosacral cerebral fluid volume. Acta Anaesthesiol Scand. 2018;62(2):234-41. https://doi.org/10.1111/aas.13046.
23. Ngan Kee WD, Khaw KS, Ng FF. Prevention of hypotension during spinal anesthesia for cesarean delivery: an effective technique using combination phenylephrine infusion and crystalloid cohydration. Anesthesiology. 2005; 103(4):744-50. https://doi.org/10.1097/00000542-200510000-00012.

24. Rout CC, Rocke DA, Levin J, Gouws E, Reddy D. A reevaluation of the role of crystalloid preload in the prevention of hypotension associated with spinal anesthesia for elective cesarean section. Anesthesiology. 1993;79(2):262-9. https://doi.org/10.1097/00000542-199308000-00011.

\section{Publisher's Note}

Springer Nature remains neutral with regard to jurisdictional claims in published maps and institutional affiliations.

\section{Ready to submit your research? Choose BMC and benefit from:}

- fast, convenient online submission

- thorough peer review by experienced researchers in your field

- rapid publication on acceptance

- support for research data, including large and complex data types

- gold Open Access which fosters wider collaboration and increased citations

- maximum visibility for your research: over $100 \mathrm{M}$ website views per year

At $\mathrm{BMC}$, research is always in progress.

Learn more biomedcentral.com/submissions 